\title{
3D micromesh-based hybrid bioprinting: multidimensional liquid patterning for 3D microtissue engineering
}

Byungjun Lee', Suryong Kim (1)', Jihoon Ko', Seung-Ryeol Lee', Youngtaek Kim', Seonghyuk Park', Jiho Kim', Sujin Hyung ${ }^{1}$, Ho-Young Kim ${ }^{1,2}$ and Noo Li Jeon (B),

\begin{abstract}
Bioprinting has been widely used to fabricate tissue engineering scaffolds and develop in vitro tissue/tumor models. Bioprinting has enabled the fabrication of complex 3D structures using different polymers and hydrogels. However, relatively low resolution and long fabrication times due to the extrusion process has resulted in limited practices for cell-based applications. Here, we present a 3D hybrid-micromesh assisted bioprinting (Hy-MAP) method that combines digital light projection (DLP) 3D-printed micromesh scaffold structures and sequential hydrogel patterning. This novel method of bioprinting enables rapid cell coculture through the allowance of various methods, including injection, dipping and draining. This approach enables the construction of mesoscale (1-50 mm) complex 3D hydrogel structures by extending the micropost-based patterning that has been demonstrated in $2 \mathrm{D}$ microfluidic channels to 3D channel networks. We established the design rules for Hy-MAP through both analytical and experimental investigations of the capillary bursting pressure (CBP) dependence on the size and geometry of the mesh as well as other physical parameters. Vascularized tumor spheroids were formed with Hy-MAP by culturing endothelial cells, stromal cell mixtures and tumor spheroids inside separate but adjacent compartments. The novel approach described in this work will provide an alternative method for fabricating mesoscale implantable tissue engineering constructs and organ-on-a-chip applications.
\end{abstract}

\section{Introduction}

Soft lithography-based microfluidic platforms have been widely used in patterning multiple cell types for tissue engineering and organ-on-a-chip applications ${ }^{1,2}$. Based on the capillary burst valve (CBV), microfluidic cell culture platforms have been actively researched by applying liquid pinning phenomena. The liquid is trapped inside the microstructure by capillary burst pressure, and the configuration of liquids can be manipulated in a predesigned shape $^{3,4}$. Liquid patterning via CBV has been researched

Correspondence: Noo Li Jeon (njeon@snu.ac.kr)

'Department of Mechanical Engineering, Seoul National University, Seoul 08826, Republic of Korea

${ }^{2}$ Institute of Advanced Machinery and Design, Seoul National University, Seoul 08826, Republic of Korea

These authors contributed equally: Byungjun Lee, Suryong Kim as follows: modification of surface hydrophilicity ${ }^{5-7}$, collocating micropost structures inside microfluidic channels to hold liquid and hydrogel/cells ${ }^{8-12}$, and utilizing a meniscus pinning barrier for barrier-free coculture ${ }^{13,14}$. However, this CBV-based organ chip is 2D-based, and it is difficult to fabricate tall structures by conventional soft lithography; thus, its use in tissue engineering applications, such as implants in the body, is difficult. To overcome these two-dimensional limitations, recent research has incorporated patterning cells in a three-dimensional way using $3 \mathrm{D}$ printing techniques ${ }^{15-17}$. In addition, research has been conducted to fabricate microlattice structures through $3 \mathrm{D}$ printing and selectively pattern fluids in three dimensions using capillary-driven flow ${ }^{18}$. With the introduction of 3D printing technology into biological research, the degree of freedom for microfluidic

\section{(c) The Author(s) 2022}

(c) (i) Open Access This article is licensed under a Creative Commons Attribution 4.0 International License, which permits use, sharing, adaptation, distribution and reproduction cc) in any medium or format, as long as you give appropriate credit to the original author(s) and the source, provide a link to the Creative Commons license, and indicate if changes were made. The images or other third party material in this article are included in the article's Creative Commons license, unless indicated otherwise in a credit line to the material. If material is not included in the article's Creative Commons license and your intended use is not permitted by statutory regulation or exceeds the permitted use, you will need to obtain permission directly from the copyright holder. To view a copy of this license, visit http://creativecommons.org/licenses/by/4.0/. 
structures has increased dramatically, and a technology called 3D bioprinting that directly locates cells and extracellular matrix (ECM) has entered the spotlight. The 3D bioprinting technique enables printing and patterning living cells and hydrogels in complex structures in three dimensions ${ }^{19-22}$ and has been diversifying and evolving in various aspects, from bioink materials and printing methods to biological applications ${ }^{23}$. For better productivity, 3D bioprinters with multiple nozzles have been developed to extrude cell-laden hydrogels and supporting materials using a single bioprinter. Supporting materials such as polycaprolactone ${ }^{24-26}$, Pluronic F-127 hydrogels $^{27,28}$, or polyethylene glycol ${ }^{29}$ have improved the poor mechanical properties of the hydrogel itself or have been utilized as a sacrificial layer. However, due to the extrusion method, bioprinting currently has limitations with respect to printing resolution and fabrication time due to the viscoelasticity of hydrogels or the size of the printing nozzle ${ }^{30-32}$.

To overcome these limitations, this study proposes a novel concept of hybrid-micromesh assisted bioprinting (Hy-MAP) that combines a high-resolution 3D printed micromesh structure and CBV-based rapid hydrogel patterning. We designed a 3D-MicroMesh Platform (3DMMP) to selectively contain liquid in designated areas using a high-resolution DLP 3D printer. In addition, we developed a technique for patterning hydrogels/cells by injecting liquid into a simple channel, a serially stacked channel or even a three-dimensionally twisted complex structure by pinning the liquid inside a mesh frame with surface tension. We have experimentally studied how bursting pressure depends on gap size and shape by increasing liquid pressure and applying it to the design of an open microfluidic chip to validate this approach. To demonstrate the advantages of open microfluidics, we not only infused liquid inside the channel by pipette but also immersed a microfluidic chip consisting of a 3D printed micromesh in fluid while gradually increasing the pressure of the fluid applied to the mesh and filling the fluid to a desired location sequentially. Furthermore, we proposed a method to form a 3D blood vessel network by patterning hydrogel/cells on 3D-MMP, showing the possibility of applying Hy-MAP to tissue engineering research in the future ${ }^{33,34}$.

\section{Materials and methods}

\section{Fabrication of the 3D-MMP and surface modification}

In this study, we carried out a study to confine liquid inside a three-dimensional micromesh. The 3D-MMP was fabricated using a commercially available high-resolution DLP 3D printer (ProJet 1200, 3D Systems). The resin used was Visjet FTX green resin (3D Systems), a mixture of triethylene glycol diacrylate (40-50 vol\%), dimethylol tricyclodecane diacrylate (15-25 vol\%), and phenylbis (2,4,6-trimethylbenzoyl) phosphine oxide (1.5-2.5 vol\%). The fabricated 3D-MMP was rinsed with isopropanol for $10 \mathrm{~min}$ and cured in a UV chamber for $30 \mathrm{~min}$. For surface modification to promote biocompatibility, a poly(p-xylylene) (Parylene) $\mathrm{C}$ coating was used with PECVD equipment (Lavida, Femto Science, Korea).

\section{Bursting pressure measurements}

Bursting pressure was measured by water-level differences with custom-made equipment. A pressure measuring device was fabricated with a 3D printer (ProJet $^{\mathrm{Tm}}$ 1200 , 3D Systems), which consisted of a hole at the bottom and a connecting luer-lock valve at the top. The luerlock valve was connected to the water tank through a long silicone tube, and the entire apparatus was filled with water free from air bubbles. By elevating the water tank at a steady speed with a step motor, the pressure working on the hole increased until the pinning liquid burst out. We recorded the process at that moment using a DSLR camera (Pentax) and analyzed the bursting moment. The bursting pressure at each gap size could be obtained by calculating the pressure applied to the fluid at the time of bursting through the recorded bursting time.

\section{Cell culture and hydrogel preparation}

Human umbilical vein endothelial cells $\left(5 \times 10^{6}\right.$ cells $\mathrm{mL}^{-1}$, HUVECs, Lonza) and human lung fibroblasts $(3 \times$ $10^{6}$ cells $\mathrm{mL}^{-1}$, LFs, Lonza) were cultured with endothelial growth medium (EGM-2, Lonza) and fibroblast growth medium (FGM-2, Lonza), respectively. U-87 MG cells used in the experiment were cultured in DMEM (HyClone, USA) supplemented with 10\% fetal bovine serum (HyClone, USA) and 1\% penicillin-streptomycin (Gibco, USA). All cells were maintained in a humidified incubator at $37^{\circ} \mathrm{C}$ and $5 \% \mathrm{CO}_{2}$. Fibrinogen $\left(2.5 \mathrm{mg} \mathrm{mL}^{-1}\right.$, Sigma Aldrich) and aprotinin $\left(0.15 \mathrm{U} \mathrm{mL}^{-1}\right.$, Sigma Aldrich) powder were dissolved in phosphate-buffered saline (HyClone), and a thrombin solution $(1 \mathrm{U} / \mathrm{mL}$, Sigma Aldrich) triggered a gelation reaction with a mixture of fibrinogen and cell suspension.

\section{Spheroid preparation}

U87MG glioblastoma multiforme (GBM) spheroids were grown in a 96-well plate with U-shaped bottom wells (Sumitomo Bakelite, Tokyo, Japan). A U87MG cell and LF suspension were prepared for a total of 5000 cells in a 1:1 ratio per well and mixed with a $1 \%$ volume ratio of Matrigel. After preculturing in the U-shaped 96-well plate for 3 days, a spheroid was introduced into the micromesh platform for vascularized tumor formation.

\section{Design of 3D-MMP for cell culture}

For the vasculogenesis experiments, micromesh platforms with three channels composed of micromesh 
arrays with a 400- $\mu \mathrm{m}$ gap and injection port $1 \mathrm{~mm}$ in size were fabricated. The three channels were all $2.6 \mathrm{~mm}$, $9.8 \mathrm{~mm}$, and $1.2 \mathrm{~mm}$ in width, length, and height, respectively, consisted of $400 \mu \mathrm{m}$ micromesh arrays and had a $1 \mathrm{~mm}$ inlet. For both the cancer vasculature experiments and tumor spheroid experiments, micromesh platforms with inner micromesh and outer micromesh structures were fabricated. The gap sizes of the inner and outer micromeshes were $400 \mu \mathrm{m}$ and $1 \mathrm{~mm}$, respectively, for the selective patterning of endothelial cells and tumor cells.

\section{Immunofluorescence microscopy image analysis}

For immunofluorescent confocal image analysis, the cell/hydrogel-embedded 3D mesh channels were washed with PBS three times and fixed with cold $4 \%$ paraformaldehyde solution for $15 \mathrm{~min}$ at room temperature. The fixed cells were permeabilized by incubation in $0.2 \%$ Triton-X (Sigma Aldrich) diluted with PBS for $15 \mathrm{~min}$ and blocked with blocking solution containing 3\% bovine serum albumin (BSA; Sigma Aldrich) in PBS to prevent nonspecific binding of antibodies for $1 \mathrm{~h}$. Then, the samples were immersed in antibody solutions and incubated for $1 \mathrm{~h}$. The antibodies used in this research were phalloidin-TRITC (1:500, Sigma Aldrich) for indicating actin filaments, FITC conjugated lectin $(25 \mu \mathrm{g} \mathrm{mL}-1$, Sigma Aldrich), FITC conjugated CD31 (1:500, ABCAM) for identifying endothelial cells, and 4'6-diamidino-2phenylindole (DAPI; 1:1,000, Sigma Aldrich) for the nucleus. Immunofluorescent images were analyzed using a FluoView FV1000 confocal laser scanning unit with an IX81 inverted microscope (Olympus) and EVOS® FL (Life Technologies) fluorescence microscope. To obtain $3 \mathrm{D}$ reconstructed images of the engineered tissues, IMARIS software (Bitplane) was used.

\section{Western blot assay}

After washing the chips with PBS, both the inner and outer regions in the chip were carefully separated, and each sample was then lysed in RIPA buffer (T\&I) containing protease inhibitors (aprotinin, leupeptin, pepstatin $\mathrm{A}$, and phenylmethylsulfonyl fluoride). The total protein concentration of the cell lysates was measured using the Bradford assay (Sigma). Lysates mixed with sample buffer were heated for $7 \mathrm{~min}$ at $95^{\circ} \mathrm{C}$, and then $10 \mu \mathrm{g}$ of protein from each sample was loaded into SDS polyacrylamide gels (8 and $12 \%$ SDS gels). After transfer to PVDF membranes (Millipore) and blocking with 5\% skim milk (Sigma) for $1.5 \mathrm{~h}$ at room temperature, the membranes were incubated overnight at $4{ }^{\circ} \mathrm{C}$ with anti-rabbit $\mathrm{CD} 31$ (1:500, Abcam), anti-mouse GFAP (1:500, Invitrogen), or anti-rabbit $\beta$-actin (1:1,000, Abcam) antibodies. After that, the membranes were washed five times with $1 \mathrm{X}$ TBST (T\&I) and incubated with goat anti-rabbit IgG
(1:1,000, Abcam) or anti-mouse IgG conjugated to horseradish peroxidase (1:1,000, Abcam) for $2 \mathrm{~h}$.

\section{Results}

Liquid trap phenomenon in the 3D micromesh structure

Liquid maintains its shape below a certain pressure limit due to the surface tension between the liquid and the solid surface of the microstructures. The micromesh structure was developed to handle the behavior of microliquids or droplets in a three-dimensional channel. The micromesh was made of photocurable polymer resin using a DLP 3D printer, and for this reason, the minimum structures that could be produced were determined. In the 3D-MMP, the thickness of the smallest rectangular frame was $200 \mu \mathrm{m}$, and the hole size varied from $400 \mu \mathrm{m}$ to $800 \mu \mathrm{m}$. Figure 1 shows an overview of the 3D micromesh channel. The liquid trapped in the structure is outwardly convex due to surface tension. In this case, the pressure acting on the fluid is determined by the radius of curvature of the surface. When the pressure applied to the liquid was smaller than the capillary busting pressure (CBP) of the micromesh, the liquid was trapped. Especially for a stationary state, the height difference between the lowest and highest level of the liquid determines the pressure exerted on the fluid according to Pascal's law. In other words, if the height difference in the vertical direction of the overall shape of the channel constituting the micromesh exceeds a certain value, the liquid cannot maintain its shape and begins to burst. Based on this design rule, Fig. 1B shows an example of this phenomenon by creating a trapezoidal cube and a Möbius band, which are three-dimensionally twisted structures with a height of $3 \mathrm{~cm}$ and a gap size of $400 \mu \mathrm{m}$. In the scanning electron microscopy image, the micromesh was generated without defects and with high accuracy. Thanks to the development of 3D printing and computer-aided design (CAD) technology, it has become possible to quickly and precisely fabricate complicated 3D structures that could not otherwise be obtained through conventional manufacturing methods such as soft lithography or injection molding. Using this $3 \mathrm{D}$ printing technology, microchannels with a complex Möbius band structure with a curved as well as a planar surface could be fabricated, and channel bundles with dimensions ranging from several hundred micrometers to several tens of millimeters can also be produced, depending on the particular applications or functions.

\section{Theoretical and experimental analysis in the liquid trap}

In this study, we applied fluid dynamics and tissue engineering cell studies by expanding the phenomenon of pinning and filling fluid in 3 dimensions. To fill the liquid in designated positions, the pressure applied to the micromesh should be below the CBP. The experimental results in Fig. 2 show that the CBP increases as the size of the hole 


\section{A}

Post-guided patterning in 2D microchannel
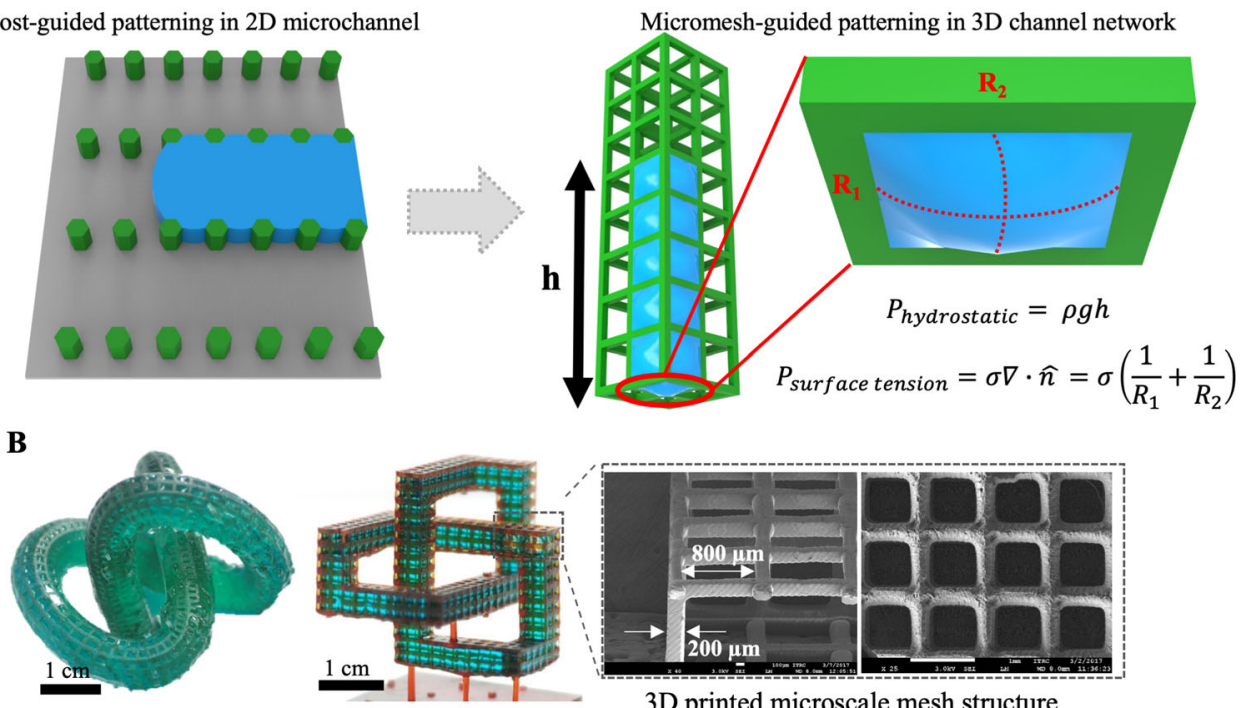

$3 \mathrm{D}$ printed microscale mesh structure

Fig. 1 Conceptual illustration of patterning liquid with the 3D printed micromesh. A Schematic explanation of the liquid patterning of postbased 2D microchannels to 3D micromesh guided patterning using Hy-MAP. The liquid was patterned in 3D mesh channel structure by surface tension. B Photograph and scanning electron microscope images of a cubic trefoil and Möbius' twisted structure, which were difficult to fabricate with conventional manufacturing methods. The size of the gap is $800 \mu \mathrm{m}$ and the thickness of the line structure is $200 \mu \mathrm{m}$.

A

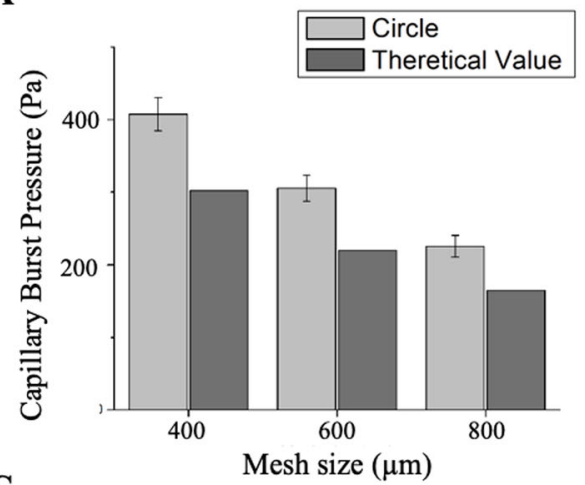

B

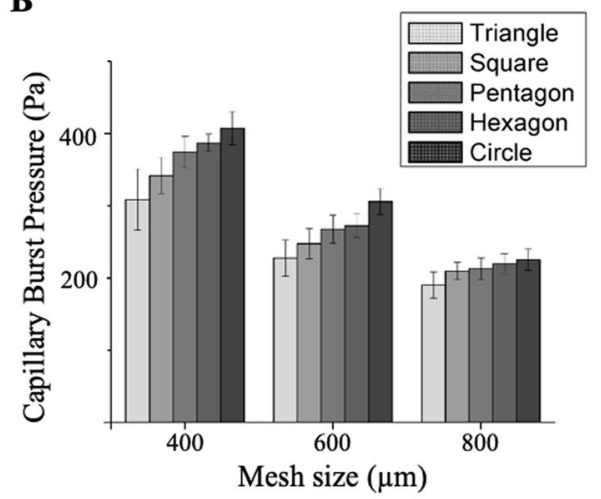

\begin{tabular}{|c|c|c|c|c|c|}
\hline Mesh type & Triangle & Square & Pentagon & Hexagon & Circle \\
\hline $\begin{array}{c}P_{\text {burst }} \sim \frac{1}{\lambda} \\
\lambda=k_{s} a \\
\text { (Equivalent circle) }\end{array}$ & & & & & \\
\hline $\begin{array}{l}\text { Shape Factor } \\
\qquad\left(k_{s}\right)\end{array}$ & 1.278 & 1.163 & 1.097 & 1.067 & 1 \\
\hline
\end{tabular}

Shape factor $(\mathrm{k})$ (Inscribed circle diameter $=\mathrm{a})$

Fig. 2 Capillary bursting pressure (CBP) as a function of shape and size of micromesh. A When the size of gap is increased from $400 \mu \mathrm{m}$ to $800 \mu \mathrm{m}$ in size, the tendency of decreasing CBP is similar in experimental and theoretical values. B CBP increases with increasing number of sides of regular polyhedral in all sizes, and it is confirmed that the effect of shape decreases as gap size increases. C Based on CBP data, shape factors ( $k$ ) were calculated for regular polygon shapes, which numerical values is used in designing other microfluidic 3D mesh structure. 
decreases and the polygon approaches the circle. Regardless of how flawless and accurate the 3D printing was, the SEM image in Fig. 1B shows that the micromesh lacked sharpness during the printing process, which caused errors between the experimental and theoretical values.

We examined how the CBP varied with the size of the gap from both analytical and experimental perspectives. For the experimental setup, we fabricated simple micromesh units of different shapes and sizes and connected them with a water reservoir. We slowly elevated the level of the water reservoir to sustain the quasi-static status and recorded the water level when the liquid burst out of the micromesh. Just before the fluid burst out of the microhole or post structure, the fluid maintained a convex shape outward in the solid-liquid-gas three-phase interface according to the Young-Laplace equation (Fig. S1). In this equation, the equilibrium pressure of the fluid could be predicted according to the two radius curvatures in the direction perpendicular to the fluid at any given point. Figure 2 shows the comparison of the calculated CBP values and the experimental values when the shape of the mesh was circular. The results show that when the gap size increased from $400 \mu \mathrm{m}$ to $800 \mu \mathrm{m}$, the CBP was observed at both the expected and measured values. A mesh structure with a gap size of $200 \mu \mathrm{m}$ or less could not be fabricated due to the resolution of the $3 \mathrm{D}$ printer used in this work. From the analytical and experimental data, it can be inferred that with a smaller gap size, a higher structure can be patterned (approximately from $20 \mathrm{~mm}$ to $40 \mathrm{~mm}$ ).

If the gap consisting of meshes is circular, the radius of curvature in both vertical directions is constant regardless of the observation direction. Therefore, it is possible to predict at what pressure the fluid will burst, as in the following equation. However, when the 3D shape of the hole or gap is a general shape other than a circle, this value is difficult to predict because the shape of the fluid just before the explosion is difficult to predict. To predict the CBP held in the polygon shape of the gap, we defined the shape factor for polygons and approximated the value through experiments. We defined a shape factor $(k)$ by comparing the CBP in a circular gap with a diameter $\boldsymbol{a}$ and CBP in a polygon gap circumscribed to this circle for shape interpolation in a regular polygon. In addition, the size of an imaginary circle corresponding to the size of each polygon was calculated and defined as lambda (equivalent diameter). Shape factor values for triangular, rectangular, pentagonal, and hexagonal gap shapes are listed in Fig. $2 \mathrm{C}$, and the values decrease as the number of sides increases.

\section{Open channel application}

Using these liquid patterning principles of micromesh, we produced a wide variety of microfluidic channels. First, a cell culture chamber with a relatively large channel size of $\sim 5 \mathrm{~mm}$ was fabricated. Despite having a relatively large amount of water and increased water pressure, the gap size of $\sim 400 \mu \mathrm{m}$ could be reliably filled because it could theoretically hold a fluid more than $30 \mathrm{~mm}$ high, as calculated from Fig. 2 above $\left(10 \mathrm{~Pa}=1 \mathrm{mmH}_{2} \mathrm{O}\right)$. By utilizing the advantage of being able to design with a $3 \mathrm{D}$ printer, we fabricated various types of microfluidic channels; for example, a microchannel with a width of $5 \mathrm{~mm}$ and a length of $20 \mathrm{~mm}$ and a cube with a $5-\mathrm{mm}$ width and 5-mm crossing cells were fabricated. Moreover, we were able to fabricate a channel with a width and height of $1 \mathrm{~mm}$ using a platform with a micromesh. A 3D microchannel with a gap size of $400 \mu \mathrm{m}$ and a frame thickness of $200 \mu \mathrm{m}$ was designed to form two individual three-dimensionally twisted channels (Fig. 3A).

Hy-MAP has the advantages of not only stably filling the fluid with the pipette but also handling the surface tension of the fluid. We proposed an application that can take advantage of open microfluidic channels. Three different channels that had gap sizes of $400 \mu \mathrm{m}, 600 \mu \mathrm{m}$, and $800 \mu \mathrm{m}$ each were composed in one plane, and the structure was gradually immersed in the water reservoir. The pressure applied to the channel gradually increased, and the three different channels were filled to different levels with water. Based on this patterning principle, we proposed a method for selective fluid patterning by dipping the whole mesh structure in a liquid or hydrogel reservoir (Fig. 3B). In conclusion, the micromesh structure-based microfluidic channel can be manufactured by choosing a size relatively freely for selective liquid patterning, and it is possible to fabricate a channel that is complicatedly entangled in three dimensions at once.

\section{Liquid patterning via a simple rotating micromesh structure}

When filling 3D-MMP, the micromesh acts as both an inlet through which the fluid can enter and a virtual wall that prevents fluid from bursting out. The liquid is captured when the hydrostatic pressure is greater than the CBP of the composing gap size, while liquid bursts out and drains if the hydrostatic pressure increases and exceeds the critical CBP of the gap size. Hydrostatic pressure is determined by the total height of the device. The effective height determined by gravitational direction can be modified by rotating the 3D-MMP with a geometrically asymmetric structure. In Fig. 4B, the length and width and height of the 3D-MMP are $40 \mathrm{~mm}, 1.4 \mathrm{~mm}$, and $17 \mathrm{~mm}$, respectively, the gap sizes of $\mathrm{S}, \mathrm{N}$, and $\mathrm{U}$ are $400 \mu \mathrm{m}$, and the gap size of the background is $1000 \mu \mathrm{m}$. When the device was dipped into liquid, the whole channel was filled with liquid (Fig. 4B-(i)). After the device was lifted from the liquid reservoir, the liquid remained in the whole channel because the hydrostatic force from $17 \mathrm{~mm}$ of height does not exceed the CBP of the $1000 \mu \mathrm{m}$ gap. However, if the device is rotated $90^{\circ}$, the height and 
A

(i)

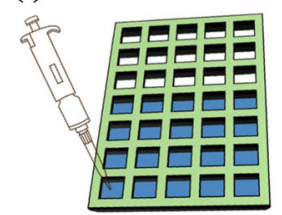

(ii)

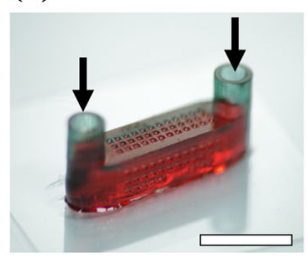

(iii)

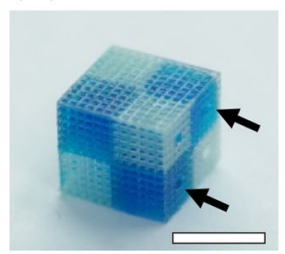

(iv)

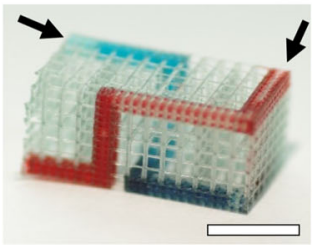

B

(i)

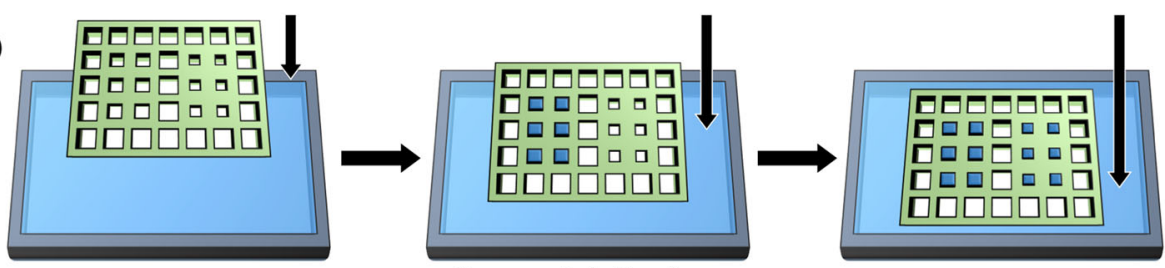

Sequential dipping

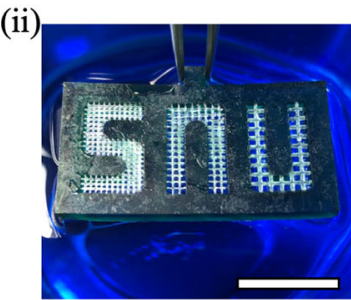

Immersed depth: $0 \mathrm{~mm}$

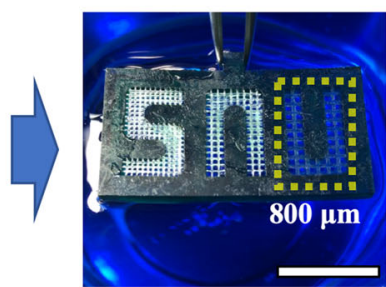

Immersed depth: $1.4 \mathrm{~mm}$

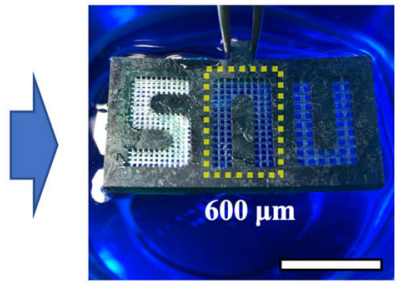

Immersed depth: $2.0 \mathrm{~mm}$

Fig. 3 Examples of trapping liquid with a micromesh structure to trap liquid in various ways. A Fabrication of microfluidic channel by injecting liquid into a micromesh and channel with width and height of $1 \mathrm{~mm}$ and a gap of $400 \mu \mathrm{m}$ to trap liquid inside the microfluidic 3D channel, threedimensionally twisted microfluidic channel (gap: $400 \mu \mathrm{m}$ and $1000 \mu \mathrm{m}$ ). Liquid inlets are marked with black arrows. B Method of selectively filling liquid by increasing the pressure applied to the mesh by gradually increasing and immersed depths when it starts to fill liquid according to the micromesh gap size. Scale bar $=1 \mathrm{~cm}$.

length of the device become interchanged. Since the hydrostatic pressure of $40 \mathrm{~mm}$ is greater than the CBP of the $1000-\mu \mathrm{m}$ gap and smaller than the CBP of $400 \mu \mathrm{m}$, the liquid in the letter(SNU) remains still, and the liquid in the background bursts out (Fig. 4B-(ii)). Using this simple dipping, rotating, and draining process, liquid can be patterned in the designated area much faster than injection through pipettes or syringes. One microliter droplet array (24) was formed inside the channel by applying this patterning method in Fig. 4C, which is expected to be applied in further multiple cell coculture or organoid research.

\section{Cell patterning and tissue engineering applications}

Various approaches toward 3D patterning of fluids and hydrogels/cells have been presented in a wide variety of ways, including two-dimensional hydrogel/cell patterning inside a microfluidic chip and 3D bioprinting techniques. In this study, we developed a technique of $3 \mathrm{D}$ patterning by trapping hydrogels and cells using a technique that can hold liquid inside a 3D micromesh structure. We suggest several liquid patterning methods: (1) direct injection, (2) serial dipping, and (3) dipping and draining, as shown in Figs. 3 and 4. This Hy-MAP method can be applied to sophisticated cell patterning and may be an alternative to $3 \mathrm{D}$ bioprinting. For the cell culture study, we fabricated a micromesh-based compartmentalized structure that enabled the selective patterning of multiple cell types. Then, the device was coated with Parylene at a height of $\sim 2 \mu \mathrm{m}$ by the oxygen plasma-enhanced CVD method to reduce the toxicity of uncured $3 \mathrm{D}$ printer resin and to ensure biocompatibility for cell culture ${ }^{35,36}$. Since 3D vasculature formation is a key element of most tissues or organs, blood vessels, and stromal cells were used. Furthermore, cancer cells or spheroids were cocultured with endothelial cells, which replicates the microphysiological system of a tumor. A cell and hydrogel mixture prior to enzymatic gelation was first filled and then cured sequentially to form a cocultured structure.

To demonstrate applications of the Hy-MAP, we performed a series of coculture models including endothelial cells, stromal cells, and cancer cells. In Figure S2, a 3D vascular network was cultured in a simple three-channel micromesh platform by injecting cell-laden fibrin gel 


\section{A}

(i) Dip

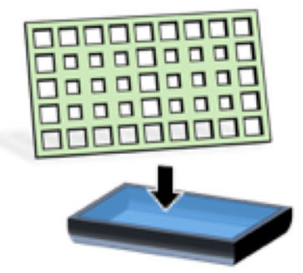

B

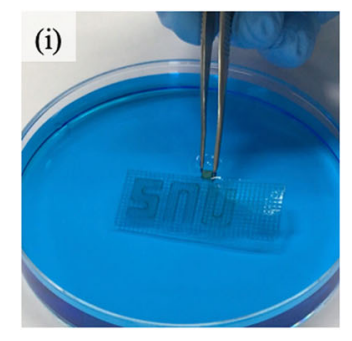

C

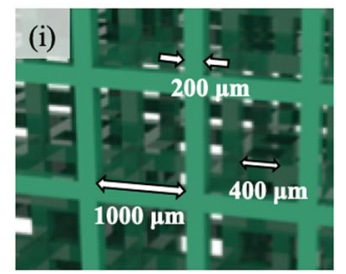

(ii) (ii) Rotate

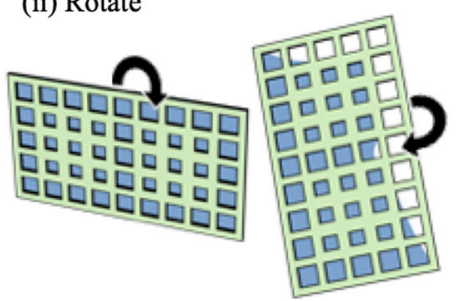

(iii) Drain
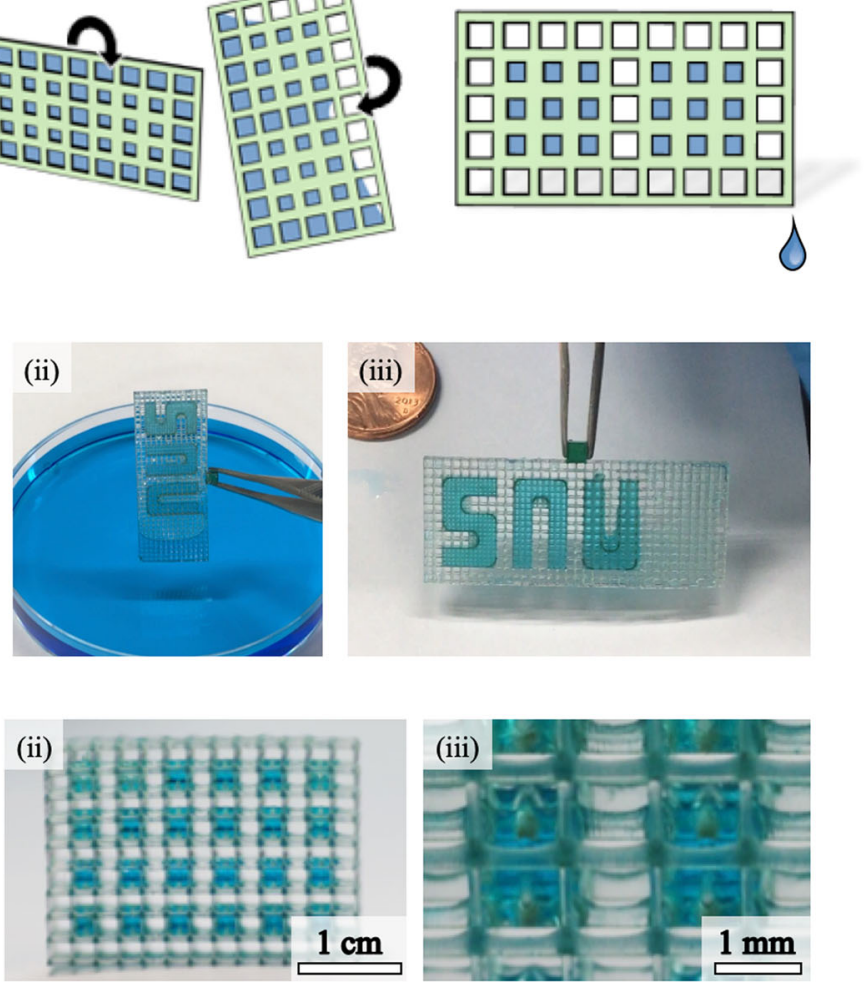

Fig. 4 Conceptual illustration and examples of liquid patterning approaches via dipping, rotating, and draining of Hy-MAP. A The process of liquid patterning. Since the effective height and length interchanges in rotation procedure, liquid bursts out except for designated area. B This patterning method was demonstrated by patterning typical words in Hy-MAP. C Liquid droplet patterning by selective draining of liquid after filling liquid in whole structure. Scale bar $=1 \mathrm{~cm}$ (ii), $1 \mathrm{~mm}$ (iii).

directly with a pipette. Then, the whole 3D-MMP was immersed in a 24-well plate with endothelial cell growth medium and cultured for 5 days. These coculture conditions were demonstrated in a previous study by our group $^{37-39}$. As shown in Figure S2, vasculogenesis was confirmed in the middle region. In the fluorescence confocal microscope photographs, red-dyed sections were stained actin filaments, and lectin was stained green. To confirm the perfusion, sectional images were analyzed to confirm that the tube had a height of 10 to $50 \mu \mathrm{m}$.

In Fig. 5, a 3D micromesh platform with two patterning areas was fabricated for tumor-vasculature coculture experiments. Two preliminary works are required for this experiment: one is a $400-500 \mu \mathrm{m}$ spheroid cultured on a U-shaped plate, and the other is a mixture of HUVECs, LFs, and fibrin gel in a 24-well plate. In the first step, tumor spheroids with $2 \mu \mathrm{l}$ of cell culture medium were introduced in the inner zone, which had a $400-\mu \mathrm{m}$ gap. Since the size of the spheroids was $>400 \mu \mathrm{m}$, the spheroids could be stably trapped in the micromesh. Then, the micromesh platforms were simply dipped into the 24-well plate, which filled the outer zone with the HUVEC, LF, and fibrin gel mixture. As can be inferred from Fig. 2, the outer zone was composed of a 1-mm gap, which theoretically can withstand $144 \mathrm{kPa}$ when it is a circle and can withstand a pressure of $123 \mathrm{kPa}$ by applying the shape factor of the square (1.163). In other words, this outer mesh could hold fluid $12.3 \mathrm{~mm}$ high on a $\mathrm{mmH}_{2} \mathrm{O}$ scale. Since the total height of the micromesh platform was $3.8 \mathrm{~mm}$, the mixture of cells and fibrin gel could stay in the micro mesh even after dipping and raising. Patterning was complete after dipping the whole platform in and out of the fibrin gel, so it was possible to perform the experiment very quickly ( $\sim 10$ platforms per minute) compared to most organ-on-a-chip or 3D bioprinting methods that directly inject or dispense the hydrogel. After 5 days of incubation, it was confirmed that blood vessels were formed as a monolayer around the spheroids and that the spheroids remained spherical in shape. In addition, compared to the control group in which only spheroids were cultured, it was confirmed that spheroids cultured alone without blood vessels hardly maintain a spherical shape but instead spread throughout the inner zone, whereas in the model cocultured with blood vessels, 


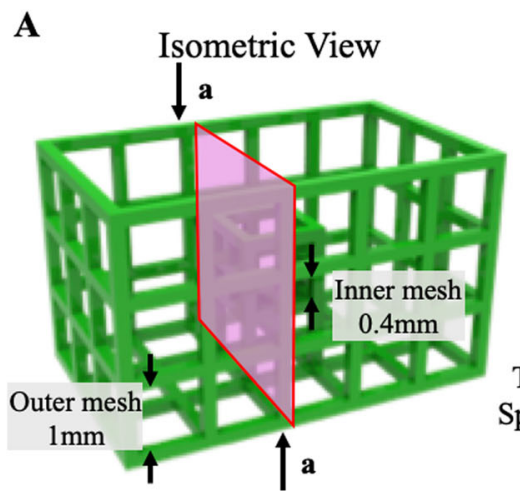

B

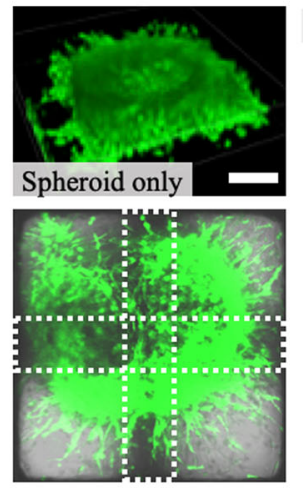

C

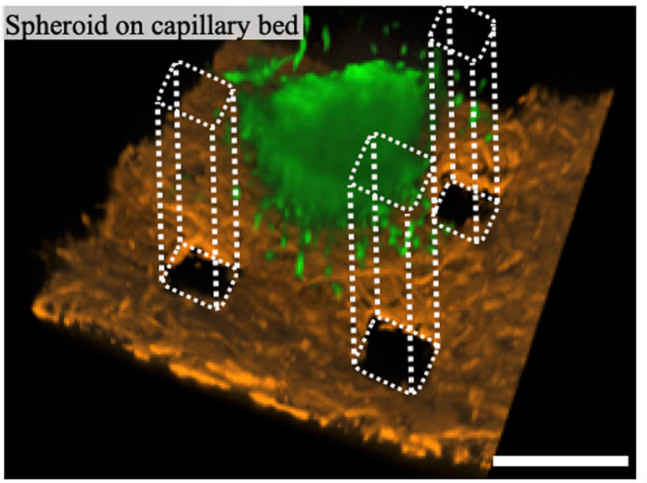

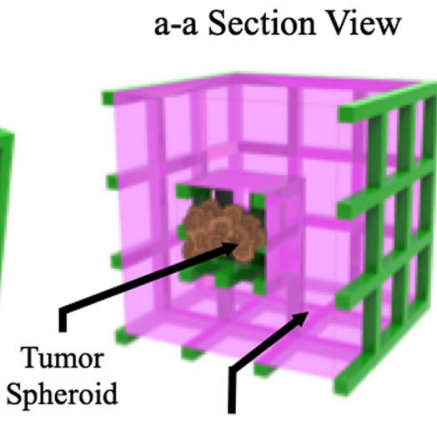

Cell-laden hydrogel

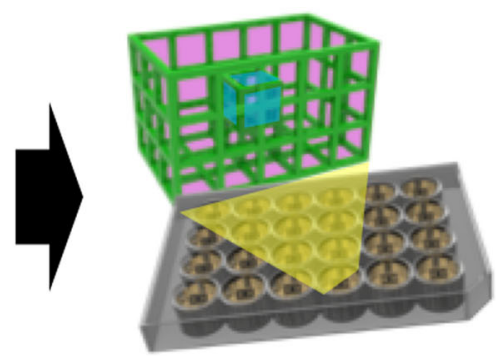

Culture for 5 days

D

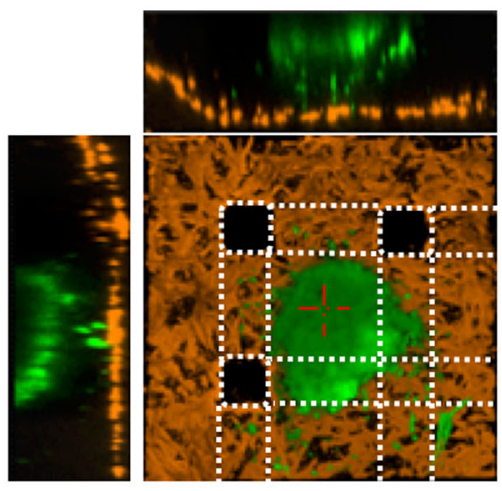

U-87 MG, GFP tagged CD31

Fig. 5 3D-MMP design for tumor-vasculature coculture model and fluorescence imaging of tumor spheroid on capillary bed. A Detailed design and patterning procedure of 3D-MMP. Cancer zone (inner mesh) consists of $400 \mu \mathrm{m}$ gap, vascular zone (outer mesh) consists of $1000 \mu \mathrm{m}$ gap. U-87 MG spheroid was placed in cancer zone by direct injection, HUVECs and LFs are patterned in vascular zone by dipping. B, C Confocal microscope images of both experimental group (HUVECs and LFs exist in the vascular zone) and control group (Only fibrin gel exists in the vascular zone) in Day 5. D Section view of vascularized tumor spheroid. Spheroid maintains spherical form on micro vascular bed. Scale bar $=200 \mu m(B)$, Scale bar $=400 \mu \mathrm{m}(\mathbf{C})$

the spheroids grew while maintaining their original shape. With this series of experiments, we demonstrated that cells could be cultured through various methods, such as direct injection, dipping, dipping and draining, and confirmed the possibility of introducing spheroids or organoids through the Hy-MAP method.

\section{Discussion}

There have been many studies that confine liquids in microstructures. The study of patterning liquids has been used in a variety of ways to pattern cells and hydrogels within microfluidics devices ${ }^{9,10,12}$. In this study, the micromesh channel structure expands in a threedimensional way by applying the phenomenon of trapping liquid inside a micromesh structure. This micromesh structure was inspired by conventional post-based 2D liquid patterning, which has been widely applied in organon-a-chip technology. We extended the post structures in three dimensions to form a micromesh structure, which enabled 3D liquid patterning. In most microfluidics, the gravitational force terms are ignored due to their small scale $(\sim 1 \mathrm{~mm})$, but since Hy-MAP is a mesoscale of a few millimeters or centimeters in size $(3-50 \mathrm{~mm})$, it is necessary to consider gravitational force ${ }^{40}$. Therefore, whether the fluid is trapped or bursts can be explained by the balance between the surface tension term acting on the fluid by the micromesh structure and the hydrostatic force term of the fluid itself. The hydrostatic pressure exerted on the fluid is determined by the height of the fluid, which can be obtained from the design of the overall structure size. In addition, we confirmed that the CBP (surface tension term) mainly depends on the shape or the size of the gap through theoretical and experimental methods. Although the experimental and theoretical values have similar tendencies, the graphs are not completely identical due to the accuracy of the $3 \mathrm{D}$ printer resolution. Furthermore, the experimental value and the analytical value deviate significantly as the gap size is smaller, as shown in Fig. 2A. This difference occurs due to equipment limitations of the DLP 3D printer, which 
reduce the effective size of the gap and the sharpness in the mesh structure. Actual printed line structures are wider than the CAD design, effectively reducing the gap between line structures. The difference between the design and actual 3D printed structure becomes more pronounced as the size of the gap decreases. In other words, if a more sophisticated 3D printer is utilized, it is expected that experimental results more similar to the theoretical values will be obtained, and experiments with a smaller gap size will be possible. If a higher resolution $3 \mathrm{D}$ printer with more accurate experimental parameters is used, the deviation between the analytical values and experimental values could be significantly smaller.

According to previous research ${ }^{3}, \mathrm{CBP}$ in micropostbased microfluidics is inversely proportional to the gap of the micropost array, and the micropost structure acts as a valve. However, this study requires a renewed interpretation method because the micromesh structure serves as a valve and the capillary burst phenomenon occurs three-dimensionally rather than two-dimensionally. We can calculate the value of the CBP of the following HyMAP studies in cell culture applications, which mainly have a rectangular gap shape. For a structure with the general shape mesh, the CBP can be easily calculated from the analytical CBP value of the circular mesh and the shape factor for each polygon, which can be applied for designing the shape and size of the gap according to the desired height of the total structure.

Micromesh is an open channel, so it is possible to pattern liquid not only by filling the liquid with an injection method such as pipette, syringe or tubing but also by utilizing the structure itself. The advantage of filling the channel by dipping the channel into a reservoir is that it can pattern liquid or hydrogel selectively and in an orderly manner without any external pump or valve by varying the size of the gap. The advantage of an open channel is that it is useful not only for confining fluid but also for removing fluid. The entire platform is immersed in a fluid that patterns the whole structure, rotated to remove all fluids other than those in a designated area, and then patterned with different types of fluids. By applying this in bioprinting, cancer cells and blood vessels can be cocultured in separate culture spaces, and it was confirmed that the cells grew in the designated area through the western blot in Fig. S3D. Since 3D vasculature formation is a key element of most tissues or organs, blood vessels and stromal cells were used. Furthermore, cancer cells and spheroids were cocultured with endothelial cells, which replicates the microphysiological system of the tumor. It is believed that this system is a suitable platform for drug permeation and reaction experiments in blood vessels because it has raised blood vessels on the open channels of the four sides.
We have suggested a new concept hybrid bioprinting system with a 3D printer and patterning method and new applications of fluid flow in terms of fluid mechanics and cell culture platforms for patterning fluids using microscale microstructures. In this study, we have overcome the limitations of conventional bioprinting systems and developed a technique for patterning liquid or hydrogel by filling liquid in a simple channel, serially stacked channel or even threedimensionally twisted complex structures by pinning liquid inside a mesh frame with surface tension. Additionally, we measured how bursting pressure depends on gap size and shape by increasing the liquid pressure and applying it to the design of the open microfluidic chip. As far as liquid pattering, we infused liquid inside the channel by both direct injection and dipping or dipping and draining mechanisms. We anticipate that this system can be applied to develop three-dimensionally organized multipart cell culture platforms for cell biology studies or drug efficacy and toxicity tests and tissue implantation for regenerative tissue engineering by adopting biodegradable micromesh materials.

\section{Acknowledgements}

This research was financially supported by the Ministry of Trade, Industry and Energy (MOTIE), and Korea Institute for Advancement of Technology (KIAT) through the International Cooperative R\&D program, Institute of Information \& Communications Technology Planning \& Evaluation (IITP) grant funded by the Korean government (MSIT) (no. 2019-0-00047), the National Research Foundation of Korea(NRF) grant funded by the Korea government(MSIT) (no.2021R1A3B1077481).

\section{Conflict of interest}

The authors declare no competing interests.

\section{Publisher's note}

Springer Nature remains neutral with regard to jurisdictional claims in published maps and institutional affiliations.

Supplementary information The online version contains supplementary material available at https://doi.org/10.1038/s41427-022-00355-x.

Received: 12 March 2021 Revised: 3 September 2021 Accepted: 27 September 2021.

Published online: 21 January 2022

\footnotetext{
References

1. Jang, K. J. et al. Fluid-shear-stress-induced translocation of aquaporin-2 and reorganization of actin cytoskeleton in renal tubular epithelial cells. Integr. Biol. 3, 134-141 (2011).

2. Chung, S. et al. Cell migration into scaffolds under co-culture conditions in a microfluidic platform. Lab Chip 9, 269-275 (2009).

3. Cho, H., Kim, H.-Y., Kang, J. Y. \& Kim, T. S. How the capillary burst microvalve works. J. Colloid Interface Sci. 306, 379-385 (2007)

4. Huang, C. P. et al. Engineering microscale cellular niches for three-dimensional multicellular co-cultures. Lab Chip 9, 1740-1748 (2009).

5. Dupuis, A., Léopoldès, J., Bucknall, D. \& Yeomans, J. Control of drop positioning using chemical patterning. Appl. Phys. Lett. 87, 024103 (2005).

6. Tian, D., Song, Y. \& Jiang, L. Patterning of controllable surface wettability for printing techniques. Chem. Soc. Rev. 42, 5184-5209 (2013).

7. Chitnis, G., Ding, Z., Chang, C.-L., Savran, C. A. \& Ziaie, B. Laser-treated hydrophobic paper: an inexpensive microfluidic platform. Lab Chip 11, 1161-1165 (2011).
} 
8. Trietsch, S. J. et al. Membrane-free culture and real-time barrier integrity assessment of perfused intestinal epithelium tubes. Nat. Commun. 8, 262 (2017).

9. Casavant, B. P. et al. Suspended microfluidics. Proc. Natl Acad. Sci. USA 110 10111-10116 (2013).

10. Berthier, J. et al. The dynamics of spontaneous capillary flow in confined and open microchannels. Sens. Transducers 183, 123 (2014).

11. Shin, Y. et al. Microfluidic assay for simultaneous culture of multiple cell types on surfaces or within hydrogels. Nat. Protoc. 7, 1247-1259 (2012).

12. Kang, M. et al. Capillarity guided patterning of microliquids. Small 11, 2789-2797 (2015).

13. van Duinen, $V$. et al. Perfused $3 D$ angiogenic sprouting in a high-throughput in vitro platform. Angiogenesis 22, 157-165 (2019).

14. Paek, J. et al. Microphysiological engineering of self-assembled and perfusable microvascular beds for the production of vascularized three-dimensional human microtissues. ACS Nano 13, 7627-7643 (2019).

15. Ligon, S. C., Liska, R., Stampfl, J., Gurr, M. \& Mulhaupt, R. Polymers for 3D printing and customized additive manufacturing. Chem. Rev. 117, 10212-10290 (2017).

16. Yang, L., Shridhar, S. V., Gerwitz, M. \& Soman, P. An in vitro vascular chip using 3D printing-enabled hydrogel casting. Biofabrication 8, 035015 (2016).

17. Grigoryan, B. et al. Multivascular networks and functional intravascular topologies within biocompatible hydrogels. Science 364, 458-464 (2019).

18. Dudukovic, N. A. et al. Cellular fluidics. Nature 595, 58-65 (2021).

19. Colosi, C. et al. Microfluidic bioprinting of heterogeneous $3 D$ tissue constructs using low-viscosity bioink. Adv. Mater. 28, 677-684 (2016).

20. Jakab, K et al. Tissue engineering by self-assembly and bio-printing of living cells. Biofabrication 2, 022001 (2010).

21. Kolesky, D. B., Homan, K. A., Skylar-Scott, M. A. \& Lewis, J. A. Three-dimensional bioprinting of thick vascularized tissues. Proc. Natl Acad. Sci. USA $\mathbf{1 1 3}$ 3179-3184 (2016)

22. Park, J. Y. et al. Development of a functional airway-on-a-chip by $3 D$ cell printing. Biofabrication 11, 15002 (2019)

23. Vanaei, S., Parizi, M., Salemizadehparizi, F. \& Vanaei, H. An overview on materials and techniques in 3d bioprinting toward biomedical application. Eng. Regen. 2, 1-18 (2021).

24. Zhang, K. L. et al. 3D bioprinting of urethra with PCL/PLCL blend and dual autologous cells in fibrin hydrogel: an in vitro evaluation of biomimetic mechanical property and cell growth environment. Acta Biomaterialia $\mathbf{5 0}$ 154-164 (2017).

25. Daly, A. C. et al. 3D bioprinting of developmentally inspired templates for whole bone organ engineering. Adv. Healthc. Mater. 5, 2353-2362 (2016).
26. Lee, H. \& Cho, D. W. One-step fabrication of an organ-on-a-chip with spatial heterogeneity using a 3D bioprinting technology. Lab Chip 16, 2618-2625 (2016).

27. Lee, J. \& Kim, G. Three-dimensional hierarchical nanofibrous collagen scaffold fabricated using fibrillated collagen and pluronic F-127 for regenerating bone tissue. ACS Appl. Mater. Interfaces 10, 35801-35811 (2018).

28. Das, S. et al. Bioprintable, cell-laden silk fibroin-gelatin hydrogel supporting multilineage differentiation of stem cells for fabrication of three-dimensional tissue constructs. Acta Biomater. 11, 233-246 (2015).

29. Shanjani, Y., Pan, C. C., Elomaa, L. \& Yang, Y. A novel bioprinting method and system for forming hybrid tissue engineering constructs. Biofabrication 7 45008 (2015).

30. Suntornnond, R. Tan, E. Y. S., An, J. \& Chua, C. K. A mathematical model on the resolution of extrusion bioprinting for the development of new bioinks. Materials 9, 756 (2016)

31. Jungst, T., Smolan, W., Schacht, K., Scheibel, T. \& Groll, J. Strategies and molecular design criteria for 3D printable hydrogels. Chem. Rev. 116, 1496-1539 (2016).

32. Knowlton, S., Onal, S., Yu, C. H., Zhao, J. J. \& Tasoglu, S. Bioprinting for cancer research. Trends Biotechnol. 33, 504-513 (2015).

33. Jeon, S., Heo, J. H., Kim, M. K., Jeong, W. \& Kang, H. W. High-precision 3D biodot printing to improve paracrine interaction between multiple types of cell spheroids. Adv. Funct. Mater. 30, 2005324 (2020).

34. $Y \mathrm{i}, \mathrm{H}$. G. et al. A bioprinted human-glioblastoma-on-a-chip for the identification of patient-specific responses to chemoradiotherapy. Nat. Biomed. Eng. $\mathbf{3}$ 509-519 (2019).

35. Song, J. S., Lee, S., Jung, S. H., Cha, G. C. \& Mun, M. S. Improved biocompatibility of parylene-C films prepared by chemical vapor deposition and the subsequent plasma treatment. J. Appl. Polym. Sci. 112, 3677-3685 (2009).

36. Chang, T. Y. et al. Cell and protein compatibility of parylene- $C$ surfaces. Langmuir 23, 11718-11725 (2007).

37. Lee, H., Kim, S., Chung, M., Kim, J. H. \& Jeon, N. L. A bioengineered array of 3D microvessels for vascular permeability assay. Microvasc. Res. 91, 90-98 (2014).

38. Ko, J. et al. Tumor spheroid-on-a-chip: a standardized microfluidic culture platform for investigating tumor angiogenesis. Lab Chip 19, 2822-2833 (2019).

39. Ko, J., Lee, Y., Lee, S., Lee, S. R. \& Jeon, N. L. Human ocular angiogenesis-inspired vascular models on an injection-molded microfluidic chip. Adv. Healthc. Mater 8, e1900328 (2019).

40. Geng, Y., Ling, S., Huang, J. \& Xu, J. Multiphase microfluidics: fundamentals, fabrication, and functions. Small 16, e1906357 (2020). 